\title{
Fuel to the Fire? Newspaper Reporting of Sexual Offending Across the US, UK, Norway, and New Zealand
}

\author{
Pål Grøndahl ${ }^{1,2}$ (D) , Ingeborg J. Sandbukt² (D) , Christine Friestad ${ }^{2,3}$ (D) , \\ Ragnar Kristoffersen ${ }^{3}$ (D) , Caitlyn P. Drinkwater ${ }^{4}$, Daniel Richardson ${ }^{4}$, \\ Gwenda M. Willis ${ }^{4}$ (D)
}

[1] Private practice, Sandvika, Norway. [2] Centre for Research and Education in Forensic Psychiatry, Oslo University Hospital, Oslo, Norway. [3] The University College of Norwegian Correctional Service, Lillestrøm, Norway. [4] School of Psychology, University of Auckland, Auckland, New Zealand.

Sexual Offending: Theory, Research, and Prevention, 2021, Vol. 16, Article e4205, https://doi.org/10.5964/sotrap.4205

Received: 2020-08-14 • Accepted: 2021-03-22 • Published (VoR): 2021-06-15

Handling Editor: Martin Rettenberger, Centre for Criminology (Kriminologische Zentralstelle - KrimZ), Wiesbaden, Germany

Corresponding Author: Pål Grøndahl, Private practice, Leif Larsensvei 4, 1338 Sandvika, Norway. E-mail: pagron50@gmail.com

\begin{abstract}
Research suggests that the news media plays an important role in shaping public opinion about sex crimes and the people who commit them, thereby influencing the development of laws and policies. The media has potential to fuel non-evidence-based policies that are ineffective and counterproductive. Alternatively, the media offers a powerful vehicle for educating the public and promoting evidence-based practices and policies. The current study aimed to examine newspaper reports of sexual crimes across four countries with different criminal justice responses to sexual offending. Constructed week sampling was used to generate samples of newspaper articles over a six-month period in 2015. Episodic articles were coded to examine how people accused/charged with sexual offenses were portrayed, the extent to which articles aligned with stereotypes, and the extent to which rehabilitation was mentioned as a solution $(n=240)$. Episodic and thematic articles were combined to code for systemic/environmental solutions to sexual crimes $(n=290)$. Overall, Norwegian articles demonstrated more neutral and less informative portrayals of individuals who have sexually offended, compared to US, UK, and New Zealand. At the same time, Norwegian articles were less likely to discuss solutions to sexual offending. Rehabilitation was rarely discussed as a solution. However, environmental/systemic solutions were discussed in approximately one third of articles. Implications for framing sexual abuse as a public health problem rather than a criminal justice problem are discussed.
\end{abstract}




\section{Keywords}

media, sexual offenses, stigma, solutions, prevention, public health

\section{Non-Technical Summary}

\section{Background}

The media occupies a prominent position in social discourse surrounding sexual offending with potential to fuel ill-informed and ineffective responses. It has been argued that the media tends to sensationalize sexual crimes by focusing on exceptional cases, contributing to an inaccurate representation of individuals convicted of sexual offenses.

\section{Why was this study done?}

We wanted to compare the newspaper coverage of sexual offending in four countries (US, UK, New Zealand, and Norway) to see how newspaper articles (i) describe the persons suspected of sexual abuse, (ii) to what degree media reporting of sexual offending aligns with common stereotypes of persons who have sexual abused (e.g., the stranger-danger notion), and (iii) to what extent any kind of solutions to the problems of sexual offending were offered.

\section{What did the researchers do and find?}

Very few of the articles had any use of sympathetic language when they described individuals charged or accused of sexual crimes. On the other hand, it was seldom that they were portrayed in pathological terms, or having an outsider status. There was a trend for US and UK articles to align more closely with stereotypes about sexual offending compared with the New Zealand and Norwegian articles.

There were few articles that mentioned individual solutions to sexual offending such as rehabilitation, which was not mentioned in any of the Norwegian articles. However, environmental/systemic solutions were discussed in approximately one third of articles.

\section{What do these findings mean?}

Encouragingly, the newspaper articles examined do not seem to add "fuel to the fire", meaning that they do not tend to sensationalize persons charged or accused of sexual crimes; however, they offered limited discussion on effective solutions to sexual offending. The media tends to frame sexual abuse as a criminal justice system problem, which is problematic because that system cannot contribute much to sexual abuse prevention efforts. Perhaps it would be more optimal to frame sexual abuse as a public health problem through which prevention is possible. 


\section{Highlights}

- The news media assumingly plays an important role in shaping public opinion about sex crimes and the people who commit them.

- Our findings do not support notions that the media is fueling the fire and sensationalizing sexual offending.

- Comparing newspaper articles from US, UK, New Zealand and Norway we found generally low rates of stigmatizing and stereotyped reporting of persons accused/ charged for sexual crimes.

- The newspaper articles offered limited discussion of effective individual solutions to sexual offending, but somewhat more on systemic solutions.

- The meagre focus on solutions on how to respond to sexual abuse might reflect that the media considers sexual crimes a criminal justice system problem to solve, rather than framing sexual abuse as a public health problem.

Sexual violence represents a serious public health problem globally (Brown, 2017; Laws, 2000; McCartan \& Gotch, 2020; McCartan et al., 2018; Shields \& Feder, 2016; WHO, 2013). According to international estimates, nearly 1 in 5 women and 1 in 10 men have experienced some form of sexual abuse during their lifetime (Pereda et al., 2009). A meta-analysis of self-reported child sexual abuse before 18 years of age indicated a prevalence of $18 \%$ among females and approximately $8 \%$ among males (Stoltenborgh et al., 2011). The negative consequences of sexual abuse are well documented (Mason \& Lodrick, 2013; Trickett et al., 2011); and since the 1990s there has been a sharp increase in public concern about individuals who have committed sexual offenses (Fox, 2013). Few other groups incite as much fear and disdain among the public as individuals with convictions for sexual offenses (McCartan, 2004; Spencer \& Ricciardelli, 2017). Public understanding of crime and justice often derives from the media (Brown et al., 2008; Sample \& Kadleck, 2008), and research suggests that the press plays an important role in shaping public opinion about sex crimes and the people who commit them (Harper \& Hogue, 2015a, 2017). Thus, the role of the media is essential with regard to how the public are informed about sexual crimes and persons who have sexually offended (Breen, 2004; Malinen et al., 2014).

It has been argued that the media sensationalize sexual crimes by focusing on exceptional cases and contribute to an inaccurate representation of individuals convicted of sexual offenses (Malinen et al., 2014; Mejia et al., 2012; Thakker \& Durrant, 2006). Furthermore, it has been claimed that the media coverage of child sexual abuse exaggerates the notion of "stranger danger" (Cheit, 2003) and that the media's presentation may intensify the perception of persons committing sex offenses as less human (Jones, 1999). Perpetrators of sexual abuse are sometimes portrayed in the media as "monsters", "beasts" or "evil perverts" who do not deserve redemption (Breen, 2004; Willis et al., 2010). These portrayals run contrary to empirical research findings. Recidivism rates 
among persons convicted of sexual crimes are in fact low, especially when it comes to new sex offenses (Hanson \& Bussière, 1998; Hanson et al., 2018). The generally low recidivism rates counter ideas such as "once an abuser always an abuser". Individuals convicted of sexual offenses represent a heterogeneous group in terms of the offenses they commit, the victims they target, their risk of reoffending and psychological characteristics (Babchishin et al., 2015; Robertiello \& Terry, 2007). With this in mind, we could ask if the media is adding fuel to the fire by painting a picture that is systematically biased.

The media's use of inflammatory language and dehumanizing labels can potentially influence public attitudes and policy in counterproductive ways (Harper et al., 2018; Willis et al., 2010). For example, Harper and Hogue (2015b) found that British readers of tabloid publications expressed much more negative perceptions about individuals who had committed sexual offenses than readers of broadsheets. The tabloid publications comprise of more negativity and hostility towards this group (Harper \& Hogue, 2015a) than the broadsheets, whose description of individuals who have committed sexual offenses tend to be more nuanced (Harper et al., 2017). The use of dehumanizing language or labels can potentially induce a negative Pygmalion effect, implying that individuals who have sexually offended start to see themselves as others see them: as inherently dangerous, morally alienated individuals undeserving of a chance at redemption. This can create barriers to community re-entry (Harper \& Hogue, 2015a; Willis et al., 2010), and unintentionally increase the very risk society wants to reduce.

Few studies have explored systematically the extent to which media reports might promote stereotypes about sexual offending and persons who have engaged in sexually abusive behavior. Mejia et al. (2012) examined US newspaper reporting of child sexual abuse in 260 articles between 2007 and 2009. Encouragingly, they found that newspaper reports did not reinforce the stranger danger stereotype. Galeste et al. (2012) explored representation of four myths in 213 US newspaper articles published in 2009. Specifically, they coded whether individuals who have offended sexually were presented as (i) compulsive, (ii) belonging to a homogenous group, (iii) "specialist" (i.e., commit exclusively sexual crimes) and (iv) untreatable. They found more than one third of articles promoted at least one stereotype with homogeneity of persons convicted for sexual offenses the most common.

Reviewing the media portrayal of sex crimes in the US across three decades from the 1980s, Mancini (2018) noted a clear association between news coverage, subsequent public concern, and the development of laws and policies. Although the causal ordering needs further investigation, Mancini (2018) pointed to the powerful impact of media exposure on directing public opinion and policy. In other words, reification of myths through the media may result in the generation and implementation of non-evidence-based policies that are ineffective or even counterproductive (Galeste et al., 2012). Alternatively, the media offers a powerful vehicle for educating the public about crime 
and justice issues, countering common myths and promoting evidence-based practices and policies. Indeed, several studies have found that education can reduce stigmatization and punitive attitudes toward individuals who have committed sexual offenses (Harper \& Hogue, 2017; King \& Roberts, 2017; Malinen et al., 2014). Moreover, research shows that referring to individuals convicted for sexual offenses in person-first language rather than stigmatizing offense-based labels such as "sex offender" contributes to more accepting public attitudes (Harris \& Socia, 2016; Lowe \& Willis, 2020). This means that how information about sexual offending is presented and expressed through campaigns to the public is also important (Kemshall \& Moulden, 2017).

In a recent review, Zatkin et al. (2021) investigated the relationship between media, public policy, and public perceptions of persons convicted of sexual crimes in the US. Based on literature published between 2007 and 2017, they concluded that there seems to be a cyclical relationship between these elements: Media coverage perpetuates institutional myths (incorrect beliefs that dictate the way the public perceives offenders about sexual offending, e.g., "stranger danger" myth), then in turn evoke the development of punitive legislation to appease the public. The public support for harsh legislation then provides a rationale for continued media coverage of the most serious cases (Zatkin et al., 2021).

A small number of studies have explored whether media reports discuss possible solutions to sexual offending. Mejia et al. (2012) found that $30 \%$ of articles discussed possible solutions, although most were concerned with addressing abuse previously perpetrated rather than preventing sexual abuse, with stronger criminal justice responses commonly suggested. Rehabilitation for persons who have committed sexual offenses was only referred to in one percent of articles coded. Similarly, in an analysis of the framing of child sexual abuse in 436 US television and newspaper reports from 2002 to 2012, law enforcement solutions were most frequently mentioned, with rehabilitation for individuals who had perpetrated sexual abuse mentioned in less than seven percent of news stories (Weatherred, 2017).

\section{International Differences in Responses to Sexual Offending}

Most research examining media reporting of sexual offending has been conducted in the US (Weatherred, 2015), a country known for its high imprisonment rate. Norway, on the other hand, is often internationally acclaimed for low rates of imprisonment, humane prison conditions and focus on rehabilitation, often referred to as an example of what is called Scandinavian Exceptionalism (Pratt, 2008). Table 1 presents the imprisonment rate (number of inmates per 100000 population) in the countries involved in the current study, as well as the proportion of the prison population imprisoned for sexual crimes. As seen, imprisonment rates differ widely, while the proportion imprisoned for sexual crimes is far less separate. 
Table 1

Number of People in Prison Per 100,000 of General Population

\begin{tabular}{|c|c|c|c|c|}
\hline Prison population & NOR $^{a}$ & $\mathrm{NZ}^{\mathrm{a}}$ & $\mathbf{U K}^{\mathbf{b}, \mathbf{c}}$ & USA $^{a}$ \\
\hline Total & 64 & 207 & 140 & 655 \\
\hline Sexual offending ${ }^{d}$ & $13(20)$ & $41(20)$ & $22(16)$ & $49(13)$ \\
\hline
\end{tabular}

a2019. Sources: National prison registers of Norway and New Zealand. USA: World Prison Brief (https:// www.prisonstudies.org) and Prison Policy Initiative (https://www.prisonpolicy.org/reports/pie2019.html).

${ }^{\text {b} W o r l d ~ P r i s o n ~ B r i e f ~(h t t p s: / / w w w . p r i s o n s t u d i e s . o r g) ~ a n d ~ E u r o s t a t ~ y e a r ~ 2016 . ~(h t t p s: / / e c . e u r o p a . e u / e u r o s t a t / w e b / ~}$ crime/data/database).

cEngland and Wales.

${ }^{\mathrm{d}}$ Numbers in parentheses show percent of prison population in prison for sex offences. US numbers only apply to state prisons.

Consistent with differences in the use of imprisonment across countries, the use of additional policies and legislation applied to individuals with sexual offense convictions varies between the countries included in this study. The US has implemented registration and community notification policies for persons convicted of sexual crimes, whereby their personal details (including photo and residential address) are maintained on a publicly accessible online register. Sexually Violent Predator (SVP) laws allow for indefinite civil commitment of persons meeting SVP commitment criteria. Commitment criteria include the presence of a specified mental disorder or mental abnormality associated with sexual recidivism risk (DeMatteo et al., 2015). Both the UK and New Zealand have registers of persons convicted of sexual crimes but these are not publicly accessible. The UK permits authorities to disclose limited information about persons with sexual offense convictions in the context of risk for serious harm, when disclosure might reasonably alleviate such risk (Blacker \& Griffin, 2010). In New Zealand Public Protection Order (PPO) legislation allows for ongoing detention of the very few individuals who have served a finite prison sentence and are considered to pose an ongoing and serious risk to the public. In January 2020, only one person was subject to a PPO (B. Raethel, personal communication, January $\left.20^{\text {th }}, 2021\right)$. Although still representing an example of relative penal moderation, Norwegian policy on sexual crimes has also changed. In 2010 stricter punishment for several types of sexual offenses was introduced in Norway (Prop. $97 \mathrm{~L}$ 2009-2010). National prison registry data shows that the average prison sentence length for persons imprisoned for sexual offenses increased from 606 days in 2010 to 867 days in 2019 , a $43 \%$ increase in nine years. Still, Norway has neither separate registers of persons convicted of sexual crimes nor any legislation allowing for detention or community supervision after final release from a prison sentence.

Contrasting criminal justice system responses to persons who have offended sexually invites questions about media reporting of sexual crimes between countries, and how media reporting might help shape evidence-based practices and policy. Given the 
complex interplay between media reporting, public opinion, and public policy, it could be hypothesized that media reporting in the US perpetuates views of persons who have sexually offended as dangerous "predators" with mental disorders. Less is known about media narratives outside the US, and whether media reports offer more balanced portrayals of persons who have sexually offended and discussions of evidenced-based approaches to their treatment and management.

\section{Study Aims}

The current study aimed to expand on extant research to explore media reporting of sexual offending across countries with contrasting criminal justice responses to sexual crimes. Existing collaborations between the researchers in Norway and New Zealand meant that Norway could be included alongside English-speaking countries. Specifically, we compared newspaper portrayals of persons suspected, accused or convicted of sexual offenses between Norway, New Zealand, the UK, and the US. The study aimed to answer the following questions and examine any cross-country differences. As the research was exploratory, few hypotheses were generated other than those stated:

1. How do newspapers portray individuals charged or accused of sexual offenses? Specifically, we examined the sex of persons reported on, whether any sympathetic language was used, and whether persons charged/accused were reported as pathological (suffering a mental disorder) or "outsiders" (originating from a different country or ethnic group to where the offending took place). We hypothesized more sympathetic language in Norwegian and NZ media reports and greater reference to mental disorder in US media reports.

2. Do newspaper reports align with common myths/stereotypes about sexual offending? Specifically, we examined the relative number of articles focused on stranger perpetrated sexual assault and recidivistic sexual offending versus sexual assaults perpetrated by individuals known to the victim/s and first-time sexual offending, respectively.

3. Do newspaper reports discuss solutions to sexual crimes? Specifically, we examined whether individual level solutions (e.g., rehabilitation) and systemic solutions (e.g., educational programs) were discussed. It was hypothesized that media reporting in countries that have dismissed publicly accessible registries altogether (i.e., Norway and New Zealand) would give greater attention to solutions than the US. 


\section{Method}

\section{Sample}

Constructed week sampling was used to replicate the normal weekly cycle of news (Luke et al., 2011) to extract samples of articles over a 6- month period. For the UK and the US articles this involved randomly selecting five Mondays from the sampling period (January 25, 2015 to July 25, 2015), and then repeating this with Tuesdays, Wednesdays and so on, until five constructed weeks including weekends were created. Using the same method twenty weeks were constructed for the New Zealand sample to approximate the sample sizes generated for the US and UK. The English language sample was selected by searching the ProQuest Newsstand database with the following key words: (sex*assault) OR (sex*violence) OR (sex*abuse) OR (sex*exploit*) OR (molest*) OR (rape) OR (sex*of$\mathrm{fen}^{*}$ ). The search strategy was applied to the full text of articles including article title and content. The sample was limited to newspaper articles from NZ, the UK and the US and printed in English.

The Norwegian sample, covering the same time period as the English language sample, was selected by searching in Retriever, a Nordic professional media monitoring agency. In order to search for comparable data in Norwegian newspapers the English key words were translated into Norwegian by two of the Norwegian authors (Grøndahl and Sandbukt). Next, two experienced Norwegian researchers (Friestad and Kristoffersen) back-translated the terms into English. A legal dictionary was also consulted. Most of the English terms that were translated into Norwegian (second parantheses) gave great unity: $\left(\operatorname{sex}^{*}\right.$ assault $)=\left(\right.$ seksu $^{*}$ overgr $\left.{ }^{*}\right),\left(\operatorname{sex}^{*}\right.$ violence $)=\left(\operatorname{seksu}^{*}\right.$ vold $),\left(\right.$ sex $^{*}$ abuse $)$


$\left(\right.$ seksu* $\left.^{*} \operatorname{lovbr}^{*}\right)$. The only term with different interpretations were $\left(\right.$ molest $\left.^{\star}\right)=\left(\right.$ overgr $\left.^{*}\right)$. The reason is likely that the Norwegian terms "overgriper/overgrep" cover more than the terms "molester/molestation", as the English language has more nuanced words in the field of sexual crimes than the Norwegian language. Overgr* was thus considered adequate. Consistent with the English samples, the search strategy was applied to the full text of all articles. After deleting duplicates and irrelevant hits, the final sample for coding consisted of 349 newspaper stories; 94 from Norway, 67 from New Zealand, 102 from the UK, and 86 from the US.

\section{Coding Protocol}

A coding protocol was developed based on the similar study by Mejia et al. (2012). Consistent with their protocol, news stories were coded for article type (article, editorial/opinion piece, letter to the editor/commentary) and reporting level (based on an event versus a thematic article with a more general focus). Several variables were considered to examine portrayals of persons accused or convicted for sexual crimes, stereotypes surrounding sexual offending, and the discussion of solutions to sexual offending. We 
considered variables coded in previous research as well as variables considered relevant to promoting evidence-based practice and policy. Ultimately, variables included were selected for ease of translation between English and Norwegian. The final protocol contained the following sections:

\section{Portrayal of Persons Charged/Accused of Sexual Offending}

This section considered characteristics of persons accused or charged with sexual offenses as reported by the media, and how those persons were described. Thematic articles (i.e., with no mention of a specific case) were not coded:

1. Sex of the individual charged or accused (female, male, both, sex not reported).

2. Whether any sympathy was portrayed for persons charged/convicted for sexual offenses (Yes or No).

3. Person accused or charged was portrayed as pathological, innately different, or inferior; for example, having physical or psychological handicaps (Yes or No).

4. Whether the individual charged or accused was described as being an outsider, i.e., coming from a country or ethnic group which is foreign to the location of offending (Yes or No).

\section{Stereotype Consistent Reporting}

This section considered the extent to which media reports were consistent with stereotypes of persons who have offended. Thematic articles (i.e., with no mention of a specific case) were not coded:

1. Person accused or charged was a stranger and unknown to the person abused, potentially promoting the "stranger danger" stereotype (Yes or No).

2. Person accused or charged had previous contact with the criminal justice system, potentially promoting perceptions of high recidivism rates (Yes or No).

\section{Solutions}

This section coded for whether any solutions were proposed as an adequate response to the content of the article. We coded for any solutions suggested or mentioned regardless of their evidence base:

1. Individual-level solutions (i.e., rehabilitation) for the person accused or charged was mentioned (Yes or No).

2. Environmental or other systemic changes were proposed. Specifically, any mention of the following options was coded:

a. Institutional/systemic prevention policies (e.g., military, foster care, university campuses, schools, and childcare).

b. Education and engagement programs (healthy sexuality and bystander outreach). 
c. Changing social determinants of health (e.g., colonialism, economic inequality, racism, homophobia, etc.).

d. Prioritizing government resources including funding.

e. Vague/unspecified calls for prevention.

f. Other (describe).

g. None.

\section{Procedure}

Approximately $10 \%$ of the English language sample $(n=28)$ and $20 \%$ of the Norwegian sample $(n=20)$ were double coded by two coders to test inter-rater reliability of the coding protocol. Discrepancies were discussed and resolved by consensus coding, and the coding protocol refined. As a rule, items were only coded as present when they were clearly present; otherwise, they were coded as absent. Remaining articles were coded by a single coder.

\section{Planned Analyses}

Data were analyzed using SPSS, version 25. Inter-rater reliability was calculated using Cohen's kappa. Frequencies for each variable of interest were calculated by country. Finally, between group comparisons of categorical data were analyzed using the Pearson chi-square test. Fisher's exact test was used when any cells had expected counts less than 5. Cramér's $V$ was used as a measure of effect size across most between-group comparisons $(d f=3, .06=$ small effect, $.17=$ medium effect, .29 = large effect; Cohen, 1988), and Cohen's $w$ was used when Cramér's $V$ was not interpretable (i.e., $d f>5$; Cohen's $w$ interpretation: .1 = small, .3 = moderate, .5 = large; Cohen, 1988).

\section{Results}

\section{Interrater Reliability of the Coding Protocol}

Cohen's Kappa statistics are reported separately for the English and Norwegian samples in Table 2. Kappa values can be interpreted as follows: 0 indicates no agreement between raters, $.01-.20$ indicates none to slight agreement, .21 - .40 indicates fair agreement, $.41-.60$ indicates moderate agreement, $.61-.80$ indicates substantial agreement and $.81-1.00$ indicates strong or almost perfect agreement. Across most variables, interrater reliability was substantial to almost perfect. A notable exception was moderate agreement for coding environmental/systemic solutions in the Norwegian sample. Given the low frequency of specific environmental solutions mentioned across all countries, the environmental/systemic solutions variable was recoded dichotomously (yes/no for any mention of an environmental/systemic solution), which increased interrater reliability for the Norwegian sample (substantial agreement). 
Table 2

Inter-Rater Reliability (Cohen's kappa) for English and Norwegian Articles

\begin{tabular}{lcc}
\hline Coding protocol section & English $^{\mathbf{a}}(\boldsymbol{n}=\mathbf{2 8})$ & Norwegian $(\boldsymbol{n}=\mathbf{2 0})$ \\
\hline Article type & 1.00 & 1.00 \\
Thematic/episodic & 1.00 & 1.00 \\
Portrayal of person accused/charged & & \\
$\quad$ Sex & 1.00 & .80 \\
Sympathy & 1.00 & 1.00 \\
Pathological & 1.00 & .90 \\
Outsider status & 1.00 & .80 \\
Stereotype consistent reporting & & .73 \\
$\quad$ Stranger & 1.00 & .90 \\
$\quad$ Repeat offending & 1.00 & \\
Solutions mentioned & & .00 \\
$\quad$ Individual & 1.00 & .44 \\
Environmental/systemic & .90 & .69 \\
$\quad$ Dichotomous coding & .86 & \\
\hline
\end{tabular}

${ }^{\mathrm{a}}$ The first $10 \%$ of all articles generated from the combined USA, UK and NZ sample.

\section{Article Types and Reporting Level}

As illustrated in Table 3, approximately two thirds of articles coded were episodic (versus thematic), reporting on a specific case. Episodic articles (i.e., without editorials and letters to the editor) were selected to examine how people accused/charged were portrayed, the extent to which media reports aligned with stereotyped views of persons who have sexually offended, and the extent to which rehabilitation was mentioned as a solution $(n=240)$. Episodic and thematic articles were combined to code for systemic/environmental solutions $(n=290)$. 
Table 3

Number of Article Types and Episodic and Thematic Reporting by Country

\begin{tabular}{|c|c|c|c|c|c|}
\hline \multirow[b]{2}{*}{ Reporting level } & \multicolumn{5}{|c|}{ Country } \\
\hline & NOR & NZ & UK & USA & Tota \\
\hline \multicolumn{6}{|c|}{ Episodic } \\
\hline \multicolumn{6}{|l|}{ Story type } \\
\hline Article & 54 & 56 & 74 & 56 & 240 \\
\hline Editorial & 1 & 1 & 2 & 5 & 9 \\
\hline Letter to editor & 0 & 0 & 1 & 1 & 2 \\
\hline Total & 55 & 57 & 77 & 62 & 251 \\
\hline \multicolumn{6}{|c|}{ Thematic } \\
\hline \multicolumn{6}{|l|}{ Story type } \\
\hline Article & 16 & 9 & 15 & 10 & 50 \\
\hline Editorial & 3 & 1 & 9 & 12 & 25 \\
\hline Letter to editor & 20 & 0 & 1 & 2 & 23 \\
\hline Total & 39 & 10 & 25 & 24 & 98 \\
\hline \multicolumn{6}{|c|}{ Total } \\
\hline \multicolumn{6}{|l|}{ Story type } \\
\hline Article & 70 & 65 & 89 & 66 & 290 \\
\hline Editorial & 4 & 2 & 11 & 17 & 34 \\
\hline Letter to editor & 20 & 0 & 2 & 3 & 25 \\
\hline Total & 94 & 67 & 102 & 86 & 349 \\
\hline
\end{tabular}

\section{Portrayal of Persons Charged or Accused of Sexual Offending}

Most persons accused or charged of sexual offenses across the sample were male $(n=$ $182 ; 75.8 \%)$. However, sex was unreported in 45 articles (18.8\%). A small number of articles from Norway $(n=4 ; 7.4 \%)$ and New Zealand $(n=7 ; 12.5 \%)$ reported on cases involving both males and females. The Norwegian sample was the only sample including articles solely concerning females charged or accused of sexual offending $(n=2 ; 3.7 \%)$, Fisher's exact test $=31.25, p<.001$, Cohen's $w=.40$. Analysis of standardized residuals revealed that Norwegian newspapers were more likely than expected to report on female perpetrated sexual offending $(z=2.3, p<.05)$, New Zealand newspapers were more likely than expected to report on cases involving both males and females $(z=2.8, p<.01)$ and UK newspapers were more likely than expected to not report on the sex of the person accused or charged for a sexual offense $(z=3.0, p<.01)$.

Individuals accused or charged with sexual offenses were seldom referred to using particularly sympathetic language; that is in 33 (13.8\%) articles. By country, New Zealand media were most likely to use sympathetic language $(n=15,26.8 \%)$ followed by the US 
$(n=8,14.3 \%)$, Norway $(n=7,13 \%)$, and the UK $(n=3,4.1 \%) ; \chi^{2}(3, n=240)=13.93, p$

$<.01$, Cramér's $V=.24$. Examination of standardized residuals revealed that New Zealand articles were significantly more likely than expected to use sympathetic language $(z=$ 2.6, $p<.01)$ and Norwegian $(z=-0.2, p<.05)$ and UK $(z=-2.2, p<.05)$ articles were significantly less likely than expected to use sympathetic language.

Few articles portrayed individuals accused or charged with sexual offenses as pathological; however, there were significant differences between countries; Fisher's exact test $=10.83, p<.01$, Cramér's $V=.20$. None of the Norwegian articles portrayed the individual accused or charged as pathological, whereas such reporting was present in $16.1 \%$ of US articles $(n=9), 10.8 \%$ of UK articles $(n=8)$, and $7.1 \%$ of NZ articles $(n=4)$. Analysis of standardized residuals revealed that the Norwegian articles were significantly less likely than expected to portray individuals charged or accused of sexual offending as pathological $(z=-2.2, p<.05)$.

Finally, the extent to which individuals accused or charged with sexual offenses were described as having an outsider status varied. In the Norwegian sample, outsider status was never reported on. In New Zealand, approximately one third of articles $(n=18$, $32.1 \%$ ) reported that the person accused or charged with a sexual offense was an outsider (i.e., from a foreign country or ethnic group), followed by the UK ( $n=18,24.3 \%)$ and US $(n=6,10.7 \%), \chi^{2}(3, n=240)=23.94, p<.001$, Cramér's $V=.32$. Examination of standardized residuals showed that reporting of outsider status was significantly more frequent than expected in New Zealand articles $(z=2.6, p<.01)$ and significantly less frequent than expected in Norwegian articles $(z=-3.1, p<.01)$.

\section{Stereotype Consistent Reporting}

Overall, stereotypes reporting was infrequent (see Table 4); however, there was a trend for US and UK articles to demonstrate more frequent stereotypes consistent reporting than the New Zealand and Norwegian articles. Chi square analysis demonstrated a significant difference in reports of stranger-perpetrated offending between countries, $\chi^{2}(3, n=240)=13.65, p=.003$, Cramér's $V=.24$; however, analysis of standardized residuals revealed no observed frequencies reached significance when compared to expected frequencies. There was no overall difference in reports of recidivistic offending between countries, $\chi^{2}(3, n=240)=2.53, p=.47$, Cramér's $V=.10$. 
Table 4

Stereotypes Reporting - By Country

\begin{tabular}{|c|c|c|c|c|c|c|c|c|c|c|}
\hline \multirow[b]{3}{*}{ Stereotype } & \multicolumn{10}{|c|}{ Country } \\
\hline & \multicolumn{2}{|c|}{ NOR } & \multicolumn{2}{|c|}{$\mathrm{NZ}$} & \multicolumn{2}{|c|}{ UK } & \multicolumn{2}{|c|}{ USA } & \multicolumn{2}{|c|}{ Total } \\
\hline & $n$ & $\%$ & $n$ & $\%$ & $n$ & $\%$ & $n$ & $\%$ & $n$ & $\%$ \\
\hline \multicolumn{11}{|l|}{ Stranger } \\
\hline No & 51 & 94.4 & 53 & 94.6 & 58 & 78.4 & 43 & 76.8 & 205 & 85.4 \\
\hline Yes & 3 & 5.6 & 3 & 5.4 & 16 & 21.6 & 13 & 23.2 & 35 & 14.6 \\
\hline Total & 54 & 100 & 56 & 100 & 74 & 100 & 56 & 100 & 240 & 100 \\
\hline \multicolumn{11}{|c|}{ Repeat offending } \\
\hline No & 49 & 90.7 & 50 & 89.3 & 62 & 83.8 & 46 & 82.1 & 207 & 86.3 \\
\hline Yes & 5 & 9.3 & 6 & 10.7 & 12 & 16.2 & 10 & 17.9 & 33 & 13.8 \\
\hline Total & 54 & 100 & 56 & 100 & 74 & 100 & 56 & 100 & 240 & 100 \\
\hline
\end{tabular}

\section{Solutions Proposed}

\section{Individual Solutions}

Individual solutions were seldom mentioned. In a total of 240 articles, 16 (7.1\%) mentioned rehabilitation. Norwegian newspapers did not present the topic at all. Rehabilitation was mentioned in eight US articles (14.3\%), six UK articles (8.1\%), and two New Zealand articles (3.6\%), Fisher's exact test $=10.07, p=.01$. Cramér's $V=.21$. Analysis of standardized residuals revealed that US newspapers were significantly more likely than expected to mention individual-level solutions $(z=2.2, p<.05)$.

\section{Environmental/Systemic Solutions}

When combining thematic and episodic articles $(n=290)$, an environmental solution was mentioned in 113 (39\%) of these. As illustrated in Table 5, institutional/systematic prevention policies were most mentioned (17\% of articles), followed by education and engagement programs (6\% of articles). Given the low frequency of specific solutions mentioned, data were recoded for the between-countries analysis to consider whether any environmental/systemic solutions were mentioned (Yes or No). Environmental or systemic solutions were more commonly mentioned in articles from New Zealand (46.2\%), the UK (46.1\%), and the US (40.9\%) than in articles from Norway $(22.9 \%), \chi^{2}(3, n=$ $290)=10.99, p=.01$, Cramér's $V=.20$. Analysis of standardized residuals confirmed that reporting environmental/systemic solutions was significantly less frequent than expected in Norwegian articles $(z=-2.2, p<.05)$. 
Table 5

Frequency of Environmental Solutions Mentioned by Country

\begin{tabular}{|c|c|c|c|c|c|c|c|c|c|c|}
\hline \multirow[b]{3}{*}{ Environmental solution mentioned } & \multicolumn{10}{|c|}{ Country } \\
\hline & \multicolumn{2}{|c|}{$\begin{array}{c}\text { NOR } \\
(n=70)\end{array}$} & \multicolumn{2}{|c|}{$\begin{array}{c}\mathrm{NZ} \\
(n=65)\end{array}$} & \multicolumn{2}{|c|}{$\begin{array}{c}\text { UK } \\
(n=89)\end{array}$} & \multicolumn{2}{|c|}{$\begin{array}{c}\text { USA } \\
(n=66)\end{array}$} & \multicolumn{2}{|c|}{$\begin{array}{c}\text { Total } \\
(n=290)\end{array}$} \\
\hline & $n$ & $\%$ & $n$ & $\%$ & $n$ & $\%$ & $n$ & $\%$ & $n$ & $\%$ \\
\hline Any solution & 16 & 22.9 & 30 & 46.2 & 41 & 46.1 & 27 & 40.9 & 113 & 39.0 \\
\hline Institutional policies & 1 & 1.4 & 13 & 20.0 & 21 & 23.6 & 14 & 21.2 & 49 & 16.9 \\
\hline Education/ engagement programs & 0 & 0.0 & 7 & 10.8 & 6 & 6.7 & 5 & 7.6 & 18 & 6.2 \\
\hline Changing social determinants and culture & 1 & 1.4 & 2 & 3.1 & 2 & 2.2 & 2 & 3.0 & 7 & 2.4 \\
\hline Resources (priority/funding) & 3 & 4.3 & 5 & 7.7 & 2 & 2.2 & 2 & 3.0 & 12 & 4.1 \\
\hline Vague/unspecified & 7 & 10.0 & 2 & 3.1 & 3 & 3.4 & 1 & 1.5 & 13 & 4.5 \\
\hline Other & 4 & 5.7 & 2 & 3.1 & 7 & 7.9 & 3 & 4.5 & 16 & 5.5 \\
\hline None & 54 & 77.1 & 35 & 53.8 & 48 & 53.9 & 39 & 59.1 & 176 & 60.7 \\
\hline
\end{tabular}

\section{Discussion}

Our aim in this paper was to expand on extant research examining media reporting of sexual crimes and explore cross-country differences in three domains of newspaper reporting. Firstly, we investigated the portrayal of individuals charged or accused of sexual crimes. The results indicated little use of sympathetic language to describe those charged/accused generally. Our hypothesis suggesting more use of sympathetic language in the least punitively oriented countries was supported only for New Zealand. However, although present in all countries except Norway, portrayals of the charged/accused in pathological terms, or based on an outsider status, were seen in a minority of articles. Secondly, we investigated stereotypes consistent reporting, which turned out to be infrequent in all countries, although somewhat more frequent in UK and US articles. Thirdly, we investigated whether solutions to sexual offending were discussed. Individual solutions were seldom mentioned, while systemic solutions were mentioned in $39 \%$ of the articles, most commonly in the UK and New Zealand articles, providing only partial support for the hypothesis of more solution-focused reporting in less punitive legal systems. It appears that the Norwegian newspapers had the least informative yet most neutral way of informing the public about sexual crimes. The coders noted that several of the Norwegian articles were remarkably short but we did not record their average length in detail. The absence of punitively oriented descriptions of sexual offenses is also interestingly contrasted to the increased penalties that have been implemented in recent years in Norway. The Norwegian articles also better represented female-perpetrated sexual offending than other countries. 
Effect sizes were generally moderate, except for a large effect found for outsider status. Interestingly, New Zealand newspaper reports were more likely than any other country to describe individuals accused or convicted of sexual offenses as coming from a foreign country or ethnic group. Such a finding might reflect New Zealand's higher proportion of foreign-born citizens relative to other countries in the current study. Specifically, national census data show that foreign-born citizens comprise approximately $27 \%$ of the New Zealand population, compared to approximately (and surprisingly similar figure) $14 \%$ of the population in Norway, the UK, and the USA. Alternatively (or additionally), such findings might reflect a "that's not us" attitude and national cohesion/pride narratives in New Zealand media.

Although stereotype consistent reporting was rare, discussion of solutions to sexual offending was not prolific. Accordingly, even though the articles sampled did not tend to promote the stranger danger and repeat offending stereotypes and therefore misinform the public, most did not educate the public about effective responses to sexual offending. Consistent with previous research (Mejia et al., 2012; Weatherred, 2017), rehabilitation was very rarely mentioned, despite its evidence for its effectiveness in reducing sexual recidivism (Gannon et al., 2019). Encouragingly, systemic solutions were discussed in approximately one third of articles, perhaps signaling a gradual shift in media reporting over time given that discussions of systemic solutions did not feature in previous studies (Mejia et al., 2012; Weatherred, 2017). Instead, solutions identified in previous research focused on law enforcement and criminal justice system approaches that target individuals. A shift towards the discussion of systemic solutions is consistent with increasing recognition of sexual abuse as a public health versus criminal justice problem and the greater potential for systemic solutions to prevent sexual offending than individual-level solutions (Tabachnick et al., 2016).

Although the primary job of the news media is to report news, how sexual abuse is framed in news reports might open or restrict opportunities to discuss effective responses. Framing sexual abuse as a public health problem invites discussions of population-level solutions, whereas framing sexual abuse as a criminal justice problem narrows the focus to individuals (Tabachnick \& Baker, 2018). Framing sexual abuse as public health problem naturally discounts stereotyped representations of persons who have abused, recognizes that sexual abuse is widespread, and highlights that all members of society can play a role in prevention. Shifting frames encourages collective responsibility for protecting potential victims from abuse and opens the door for people at risk to abuse to seek help before they cause harm (e.g., Malone, 2016). Put simply, framing sexual abuse as a public health problem (like alcohol/drug impaired driving and HIV transmission) communicates that prevention is possible and therefore invites proactive discussion about systemic/environmental solutions to sexual offending. Such solutions range from prevention education in schools and institutional prevention policies through prevention programs for individuals at risk of sexual offending and influencing social determinants 
of health. As highlighted in the current study, of all environmental/systemic solutions considered, institutional prevention policies were most discussed. One implication for professionals working in the field of sexual abuse treatment and prevention is to use media opportunities that might follow news reports about an individual case to discuss broad community and population-level prevention strategies.

\section{Limitations and Implications for Future Research}

Several limitations of the current study must be acknowledged. We utilized samples of newspaper articles from a six-month period in 2015, and it is uncertain whether findings could be generalized beyond that period. It could be argued that the sample is somewhat outdated and before the \#meToo movement occurred, which undoubtedly influenced media reporting. However, the articles sampled were more recent than samples used in previous studies (e.g., Galeste et al., 2012; Mejia et al., 2012; Weatherred, 2017), and findings therefore offer an independent contribution to extant research on newspaper reporting of sexual offending. We focused on news articles rather than editorials or letters to the editor, which reduced already small sample sizes for each country. On the other hand, sample sizes were generally sufficient to detect significant between-group differences which corresponded to moderate effect sizes. Small sample sizes might have explained the failure to detect significant differences between observed and expected frequencies for stereotypes consistent reporting across countries. A focus on newspaper articles represents another limitation given the fact that news is commonly accessed from alternative sources including social media. In addition, newspapers were not further separated into broadsheet or tabloid journalism. A clear implication for future research is to identify the most accessed and trusted news sources from which to examine media narratives.

The coding protocol was not without limitations. In retrospect, the item considering whether any sympathy was portrayed for persons charged/convicted for sexual offenses departed from the initial intent of coding for humane and respectful reporting of individuals responsible for sexual crimes (e.g., use of person-first language) rather than reliance on offense-based and other stigmatizing labels. In addition, the coding protocol was limited to items that could be rated reliably and translated readily from English to Norwegian. There will always be challenges associated with translating legal and criminological terminology from one language to another and back again, and this might be reflected in the slightly lower interrater reliability obtained between raters for the Norwegian versus English newspapers. Some of the English terms have several possible translations in Norwegian, and vice versa. For these reasons, it is important in future research utilizing samples of articles in different languages that search terms and translations are as accurate as possible to include all versions of a term or concept. In addition, to compare media reporting across broad domains and between countries, the coding protocol did not consider cultural nuances in media reporting specific to each 
country. Finally, some articles may have been overlooked due to missing key words in our search strategy (e.g., pedophile/paedophile, or predator*).

\section{Conclusions}

The media occupies a prominent position in social discourse surrounding sexual offending with potential to fuel ill-informed and ineffective responses. Alternatively, the media has potential to drive evidence-based responses. Encouragingly, we found generally low rates of stigmatizing and stereotyped reporting of sexual crimes across newspaper articles examined. Findings do not support notions that the media is fueling the fire and sensationalizing sexual offending - possibly reflecting shifting media narratives especially in the wake of high-profile celebrity cases (e.g., Jimmy Savile, Gary Glitter), which contradict the stranger danger and repeat offending stereotypes. At the same time, newspaper articles offered limited discussion of effective solutions to sexual offending, which possibly reflects media framing of sexual abuse as an issue for the criminal justice system to solve, rather than framing sexual abuse as a preventable public health problem.

Funding: The authors have no funding to report.

Acknowledgments: The authors have no additional (i.e., non-financial) support to report.

Competing Interests: The authors have declared that no competing interests exist.

Data Availability: The data can be used by colleagues upon request to the corresponding author.

\section{References}

Babchishin, K. M., Hanson, R. K., \& VanZuylen, H. (2015). Online child pornography offenders are different: A meta-analysis of the characteristics of online and offline sex offenders against children. Archives of Sexual Behavior, 44(1), 45-66. https://doi.org/10.1007/s10508-014-0270-x

Blacker, K., \& Griffin, L. (2010). Megan's Law and Sarah's Law: A comparative study of sex offender community notification schemes in the United States and the United Kingdom. Criminal Law Bulletin, 46, 987-1009. http://digitalcommons.pace.edu/lawfaculty/750

Breen, M. J. (2004). 'Depraved paedos and other beasts': The media portrayal of child sexual abusers in Ireland and the UK. In P. Yonder \& P. M. Kreuter (Eds.), Monsters and the monstrous: Myths and metaphors of enduring evil (pp. 285-291). Interdisciplinary Press.

Brown, J. (2017). Public health, prevention and risk management. In K. McCartan \& H. Kemshall (Eds.), Contemporary sex offender risk management: Volume 1. Perceptions (pp. 35-60). Springer. https://doi.org/10.1007/978-3-319-63567-5_2 
Brown, S., Deakin, J., \& Spencer, J. (2008). What people think about the management of sex offenders in the community. Howard fournal of Criminal fustice, 47(3), 259-274. https://doi.org/10.1111/j.1468-2311.2008.00519.x

Cheit, R. E. (2003). What hysteria? A systematic study of newspaper coverage of accused child molesters. Child Abuse \& Neglect, 27(6), 607-623. https://doi.org/10.1016/S0145-2134(03)00108-X

Cohen, J. (1988). Statistical power analysis for the behavioral sciences. Routledge Academic.

DeMatteo, D., Murphy, M., Galloway, M., \& Krauss, D. A. (2015). A national survey of United States sexually violent person legislation: Policy, procedures, and practice. International fournal of Forensic Mental Health, 14(4), 245-266. https://doi.org/10.1080/14999013.2015.1110847

Fox, K. J. (2013). Incurable sex offenders, lousy judges \& the media: Moral panic sustenance in the age of new media. American fournal of Criminal fustice, 38(1), 160-181.

https://doi.org/10.1007/s12103-012-9154-6

Galeste, M. A., Fradella, H. F., \& Vogel, B. (2012). Sex offender myths in print media: Separating fact from fiction in U.S. newspapers. Western Criminology Review, 13(2), 4-24. https:/www.westerncriminology.org/documents/WCR/v13n2/v13n2.pdf

Gannon, T. A., Olver, M. E., Mallion, J. S., \& James, M. (2019). Does specialized psychological treatment for offending reduce recidivism? A meta-analysis examining staff and program variables as predictors of treatment effectiveness. Clinical Psychology Review, 73, Article 101752. https://doi.org/10.1016/j.cpr.2019.101752

Hanson, R. K., \& Bussière, M. T. (1998). Predicting relapse: A meta-analysis of sexual offender recidivism studies. Journal of Consulting and Clinical Psychology, 66(2), 348-362.

https://doi.org/10.1037/0022-006X.66.2.348

Hanson, R. K., Harris, A. J. R., Letourneau, E., Helmus, L. M., \& Thornton, D. (2018). Reductions in risk based on time offense-free in the community: Once a sexual offender, not always a sexual offender. Psychology, Public Policy, and Law, 24(1), 48-63. https://doi.org/10.1037/law0000135

Harper, C. A., Bartels, R. M., \& Hogue, T. E. (2018). Reducing stigma and punitive attitudes toward pedophiles through narrative humanization. Sexual Abuse, 30(5), 533-555.

https://doi.org/10.1177/1079063216681561

Harper, C. A., \& Hogue, T. E. (2015a). The emotional representation of sexual crime in the national British press. Fournal of Language and Social Psychology, 34(1), 3-24. https://doi.org/10.1177/0261927X14544474

Harper, C. A., \& Hogue, T. E. (2015b). Measuring public perceptions of sex offenders: Reimagining the Community Attitudes Toward Sex Offenders (CATSO) scale. Psychology, Crime \& Law, 21(5), 452-470. https://doi.org/10.1080/1068316X.2014.989170

Harper, C. A., \& Hogue, T. E. (2017). Press coverage as a heuristic guide for social decision-making about sexual offenders. Psychology, Crime \& Law, 23(2), 118-134. https://doi.org/10.1080/1068316X.2016.1227816

Harper, C. A., Hogue, T. E., \& Bartels, R. M. (2017). Attitudes towards sexual offenders: What do we know, and why are they important? Aggression and Violent Behavior, 34, 201-213. https://doi.org/10.1016/j.avb.2017.01.011 
Harris, A. J., \& Socia, K. M. (2016). What's in a name? Evaluating the effects of the "sex offender" label on public opinions and beliefs. Sexual Abuse, 28(7), 660-678. https://doi.org/10.1177/1079063214564391

Jones, K. D. (1999). The media and megan's law: Is community notification the answer? The fournal of Humanistic Counseling, Education and Development, 38(2), 80-88. https://doi.org/10.1002/j.2164-490X.1999.tb00066.x

Kemshall, H., \& Moulden, H. M. (2017). Communicating about child sexual abuse with the public: Learning the lessons from public awareness campaigns. Fournal of Sexual Aggression, 23(2), 124-138. https://doi.org/10.1080/13552600.2016.1222004

King, L. L., \& Roberts, J. J. (2017). The complexity of public attitudes toward sex crimes. Victims \& Offenders, 12(1), 71-89. https://doi.org/10.1080/15564886.2015.1005266

Laws, D. R. (2000). Sexual offending as a public health problem: A North American perspective. Journal of Sexual Aggression, 5(1), 30-44. https://doi.org/10.1080/13552600008413294

Lowe, G., \& Willis, G. (2020). "Sex offender” versus "person”: The influence of labels on willingness to volunteer with people who have sexually abused. Sexual Abuse, 32(5), 591-613. https://doi.org/10.1177/1079063219841904

Luke, D. A., Caburnay, C. A., \& Cohen, E. L. (2011). How much is enough? New recommendations for using constructed week sampling in newspaper content analysis of health stories. Communication Methods and Measures, 5(1), 76-91. https://doi.org/10.1080/19312458.2010.547823

Malinen, S., Willis, G. M., \& Johnston, L. (2014). 2014/07/03). Might informative media reporting of sexual offending influence community members' attitudes towards sex offenders? Psychology, Crime \& Law, 20(6), 535-552. https://doi.org/10.1080/1068316X.2013.793770

Malone, L. (2016). Help wanted: Young pedophiles and the importance of primary prevention. In E. Jeglic \& C. Calkins (Eds.), Sexual violence (pp. 273-283). Springer. https://doi.org/10.1007/978-3-319-44504-5_15

Mancini, C. (2018). The media, public opinion and sex offender policy in the U.S. In P. Lussier \& E. Beauregard (Eds.), Sexual offending - A criminological perspective (pp. 116-137). Routledge.

Mason, F., \& Lodrick, Z. (2013). Psychological consequences of sexual assault. Best Practice \& Research Clinical Obstetrics \& Gynaecology, 27(1), 27-37.

https://doi.org/10.1016/j.bpobgyn.2012.08.015

McCartan, K. (2004). 'Here there be monsters': The public's perception of paedophiles with particular reference to Belfast and Leicester. Medicine, Science, and the Law, 44(4), 327-342. https://doi.org/10.1258/rsmmsl.44.4.327

McCartan, K., \& Gotch, K. (2020). International approaches to the management of perpetrators of sexual harm policy. In J. Proulx, F. Cortoni, L. A. Craig, \& E. J. Letourneau (Eds.), The Wiley handbook of what works with sexual offenders (pp. 441-454). Wiley. https://doi.org/10.1002/9781119439325.ch25

McCartan, K. F., Merdian, H. L., Perkins, D. E., \& Kettleborough, D. (2018). Ethics and issues of secondary prevention efforts in child sexual abuse. International fournal of Offender Therapy and Comparative Criminology, 62(9), 2548-2566. https://doi.org/10.1177/0306624X17723951 
Mejia, P., Cheyne, A., \& Dorfman, L. (2012). News coverage of child sexual abuse and prevention, 2007-2009. Journal of Child Sexual Abuse, 21(4), 470-487. https://doi.org/10.1080/10538712.2012.692465

Pereda, N., Guilera, G., Forns, M., \& Gómez-Benito, J. (2009, June). The prevalence of child sexual abuse in community and student samples: A meta-analysis. Clinical Psychology Review, 29(4), 328-338. https://doi.org/10.1016/j.cpr.2009.02.007

Pratt, J. (2008). Scandinavian exceptionalism in an era of penal excess: Part II: Does Scandinavian exceptionalism have a future? British fournal of Criminology, 48(3), 275-292. https://doi.org/10.1093/bjc/azm073

Robertiello, G., \& Terry, K. J. (2007). Can we profile sex offenders? A review of sex offender typologies. Aggression and Violent Behavior, 12(5), 508-518. https://doi.org/10.1016/j.avb.2007.02.010

Sample, L. L., \& Kadleck, C. (2008). Sex offender laws: Legislators' accounts of the need for policy. Criminal fustice Policy Review, 19, 40-62. https://doi.org/10.1177/0887403407308292

Shields, R. T., \& Feder, K. A. (2016). The public health approach to preventing sexual violence. In E. L. Jeglic \& C. Calkins (Eds.), Sexual violence: Evidence based policy and prevention (pp. 129-144). Springer. https://doi.org/10.1007/978-3-319-44504-5_9

Spencer, D., \& Ricciardelli, R. (2017). 'They're a very sick group of individuals': Correctional officers, emotions, and sex offenders. Theoretical Criminology, 21(3), 380-394. https://doi.org/10.1177/1362480616647590

Stoltenborgh, M., van Ijzendoorn, M. H., Euser, E. M., \& Bakermans-Kranenburg, M. J. (2011). A global perspective on child sexual abuse: Meta-analysis of prevalence around the world. Child Maltreatment, 16(2), 79-101. https://doi.org/10.1177/1077559511403920

Tabachnick, J., \& Baker, K. (2018). Engaging the public through messaging and bystander actions. In M. Erooga (Ed.), Protecting children and adults from abuse after Savile: what organisations and institutions need to do (pp. 163-185). Jessica Kingsley Publishers.

Tabachnick, J., McCartan, K., \& Panaro, R. (2016). Changing course: From a victim/offender duality to a public health perspective. In D. Laws \& W. O'Donohue (Eds.), Treatment of sex offenders (pp. 323-342). Springer. https://doi.org/10.1007/978-3-319-25868-3_14

Thakker, J. D., \& Durrant, R. (2006). News coverage of sexual offending in New Zealand, 2003. New Zealand fournal of Psychology, 35(1), 28-35.

Trickett, P. K., Noll, J. G., \& Putnam, F. W. (2011). The impact of sexual abuse on female development: Lessons from a multigenerational, longitudinal research study. Development and Psychopathology, 23(2), 453-476. https://doi.org/10.1017/S0954579411000174

Weatherred, J. L. (2015). Child sexual abuse and the media: A literature review. Fournal of Child Sexual Abuse, 24(1), 16-34. https://doi.org/10.1080/10538712.2015.976302

Weatherred, J. L. (2017). Framing child sexual abuse: A longitudinal content analysis of newspaper and television coverage, 2002-2012. Journal of Child Sexual Abuse, 26(1), 3-22.

https://doi.org/10.1080/10538712.2016.1257528 
WHO. (2013). Global and regional estimates of violence against women: Prevalence and health effects of intimate partner violence and non-partner sexual violence.

https://www.who.int/publications/i/item/9789241564625

Willis, G. M., Levenson, J. S., \& Ward, T. (2010). Desistance and attitudes towards sex offenders: Facilitation or hindrance? Journal of Family Violence, 25(6), 545-556.

https://doi.org/10.1007/s10896-010-9314-8

Zatkin, J., Sitney, M., \& Kaufman, K. (2021). The relationship between policy, media, and perceptions of sexual offenders between 2007 and 2017: A review of the literature. Trauma, Violence \& Abuse. Advance online publication. https://doi.org/10.1177/1524838020985568

IATSO=-

International Association for the
Ireatment of Sexual Offenders

Sexual Offending: Theory, Research, and Prevention (SOTRAP) is the official journal of the International Association for the Treatment of Sexual Offenders (IATSO).
(P) leibniz-psychology.org

PsychOpen GOLD is a publishing service by Leibniz Institute for Psychology (ZPID), Germany. 